\title{
Validated Alzheimer's Disease Risk Index (ANU-ADRI) is associated with smaller volumes in the default mode network in the early $60 \mathrm{~s}$
}

\author{
Nicolas Cherbuin ${ }^{1}\left[\right.$ - Marnie E. Shaw ${ }^{1} \cdot$ Erin Walsh $^{1} \cdot$ Perminder Sachdev ${ }^{2} \cdot$ Kaarin J. Anstey $^{1}$
}

Published online: 14 December 2017

(c) The Author(s) 2017. This article is an open access publication

\begin{abstract}
Strong evidence is available suggesting that effective reduction of exposure to demonstrated modifiable risk factors in midlife or before could significantly decrease the incidence of Alzheimer's disease (AD) and delay its onset. A key ingredient to achieving this goal is the reliable identification of individuals at risk well before they develop clinical symptoms. The aim of this study was to provide further neuroimaging evidence of the effectiveness of a validated tool, the ANU Alzheimer's Disease Risk Index, for the assessment of future risk of cognitive decline. Participants were 461 (60-64 years, 48\% female) community-living individuals free of dementia at baseline. Associations between risk estimates obtained with the ANUADRI, total and regional brain volumes including in the default mode network (DMN) measured at the same assessment and diagnosis of MCI/dementia over a 12-year follow-up were tested in a large sample of community-living individuals free of dementia at baseline. Higher risk estimates on the ANU-ADRI were associated with lower cortical gray matter and particularly in the DMN. Importantly, difference in participants with high and low risk scores explained 7-9\% of the observed difference in gray matter volume. In this sample, every one additional risk point on the ANU-ADRI was associated with an $8 \%$ increased risk of developing MCI/dementia over a 12-year follow-up and this association was partly mediated by a subregion of the DMN. Risk of cognitive decline assessed with a validated instrument is associated with gray matter volume, particularly in the DMN, a region known to be implicated in the pathological process of the disease.
\end{abstract}

Keywords Dementia risk $\cdot$ ANU-ADRI $\cdot \mathrm{DMN} \cdot \mathrm{MRI} \cdot \mathrm{MCI}$

\section{Introduction}

In the context of population ageing, Alzheimer's disease (AD) is becoming an unsustainable burden at the individual, social and economic levels. Although investment in dementia research has increased very substantially in recent times, current evidence suggests that a disease modifying treatment is unlikely to be available in the clinic before a decade or more. Moreover, if and when such treatment becomes available, due to the multi-factorial nature of the disease, it will be unlikely to fully cure the complex underlying pathology or to be widely available or affordable in a timely fashion.

Nicolas Cherbuin

nicolas.cherbuin@anu.edu.au

1 Centre for Research on Ageing, Health and Wellbeing, Australian National University, 54 Mills Road, Canberra, ACT 2601, Australia

2 School of Psychiatry, University of New South Wales, Sydney, Australia
In addition, it is now increasingly recognised that individuals in the pre-symptomatic stages and who have not already suffered extensive neurodegeneration are likely to benefit most from any pharmacological or non-pharmacological treatment.

Consequently, it is essential to develop strategies to identify cognitively healthy individuals who are at particular risk of developing $\mathrm{AD}$ in order to better target future treatments as they become available, and in the meantime, to implement preventative strategies aimed at decreasing risk in the population. This is particularly relevant as evidence confirming the contribution of modifiable risk factors to the disease process, and the effectiveness of interventions aimed at decreasing risk exposure is becoming increasingly strong (Xu et al. 2015).

With this in mind, we have developed a reliable evidencebased AD risk assessment instrument, called the Australian National University Alzheimer Disease Risk Index (ANUADRI), that does not require complex or costly clinical tests and which can be administered face-to-face or online 
(Anstey et al. 2013). It relies on robust estimates from highquality published systematic reviews summarising the effect of fifteen established AD risk factors including age, sex, education, diabetes, body mass index (BMI), hypercholesterolemia, stroke, traumatic brain injury, depression, physical activity, smoking, dietary fish intake, alcohol consumption, pesticide exposure, cognitive and social engagement. Importantly, the ANU-ADRI has been externally validated in three large international cohort studies and was found to be reliable in predicting prospectively the development of Alzheimer's disease, and was also found to be robust to the inclusion of an incomplete set of risk measures if all are not available (Anstey et al. 2014). In a recent study, we also demonstrated that higher scores on the ANU-ADRI were predictive of conversion to mild cognitive impairment (MCI) (Andrews et al. 2017).

The aim of the present study was to further build the evidence supporting the validity of this instrument by demonstrating a cross-sectional association between ANU-ADRI scores and individual variation in total brain volume (TBV), cortical gray matter (GM) volume, hippocampal volume (HC), and the volume of the structures of the brain default mode network (DMN). The DMN is particularly relevant in this context because it has been shown to be consistently affected by AD-related neurodegeneration relatively early in the disease process (Simic et al. 2014; Petrella et al. 2011; Fjell et al. 2014; Mormino et al. 1991). For example, amyloid plaque deposition assessed with PIB-PET is higher in the DMN and occurs concurrently to hypometabolism which also develops preferentially in this region (Grothe and Teipel 2016). The DMN is also one of the brain networks most affected by gray matter atrophy (Grothe and Teipel 2016; Tondelli et al. 2012). Importantly, these changes in the DMN are detectable well before (10 years or more) clinical diagnoses of MCI or AD are established in affected individuals (Tondelli et al. 2012; Sheline et al. 2010).

Thus, we predicted that higher ANU-ADRI scores in individuals participating in a large community-based study of ageing would be associated with lower total and region of interest (ROI) volumes. In addition, we hypothesised that risk scores and brain volumes related to risk levels would be predictive of conversion to $\mathrm{MCI} /$ dementia over a 12 year follow-up.

\section{Methods}

\section{Study population}

Participants included in the present study were selected from the larger PATH Through Life (PATH) project which has been described elsewhere (Anstey et al. 2012). Briefly, PATH randomly sampled individuals from the electoral roll of the city of Canberra and adjoining town of Queanbeyan across three age groups. The focus of this investigation is on the older cohort $(n=2551)$ aged $60-64$ at baseline in 2001. Participants were included if they had undergone a brain scan at the first assessment $(n=551$, wave 1$)$ and met inclusion criteria. After excluding those with a MMSE $<25$ $(n=29)$, stroke $(n=14)$, epilepsy $(n=2)$, or Parkinson's disease $(n=10), 496$ participants were available for analyses. A further 35 participants were excluded due to failed neuroimaging processing quality control criteria leaving 461 for further analysis. Compared to those excluded, included participants did not differ in age and sex but had a somewhat higher level of education (13.99 vs 13.71 years) and higher scores on the MMSE (29.34 vs 29.04).

\section{Standard protocol approvals, registrations, and patient consent}

The study was approved by the Australian National University Ethics Committee and all participants provided written informed consent.

\section{Socio-demographic and health measures}

Total years of education, stroke, depression symptomatology (Goldberg depression) (Goldberg et al. 1988), and smoking (ever) were assessed by self-report. Body mass index (BMI) was computed with the formula weight $(\mathrm{kg}) / \mathrm{height} \mathrm{x}$ height $\left(\mathrm{m}^{2}\right)$ based on self-report of weight and height. Systolic and diastolic blood pressures were computed over two measurements using an Omron M4 monitor after a rest of at least $5 \mathrm{~min}$. Participants were classified as hypertensive if their mean systolic or diastolic blood pressure measures were higher than 140 and $90 \mathrm{mmHg}$ respectively or if they took anti-hypertensive medication. APO*E4 genotype was determined based on buccal swabs using QIAGEN DNA Blood kits (\#51162; QIAGEN, Hilden, Germany). Participants were classified as APO*E4 carriers if they possessed one or two $\varepsilon 4$ alleles and as non-carriers otherwise.

\section{MRI scan acquisition and image analysis}

All participants were imaged with a 1.5 T Philips Gyroscan ACS-NT scanner (Philips Medical Systems, Best, The Netherlands) for T1-weighted 3-D structural scan (fast-field echo sequence $\left[\mathrm{TR} / \mathrm{TE} / \mathrm{FA}=28.05 / 2.64 \mathrm{~ms} / 30^{\circ}\right]$ matrix size $=256 \times 256$; field of view $=260$, for an in plane resolution of $1 \mathrm{~mm} \times 1 \mathrm{~mm}$ ). All images were pre-processed using the MINC imaging toolbox (MINC; http://en.wikibooks. org/wiki/MINC) which included image intensity normalisation and $B_{0}$ inhomogeneity correction (Sled et al. 1998). Further image analysis was carried out using FreeSurfer (v. 5.3) (Fischl 2012). The image processing steps consist of 
motion correction and averaging, removal of non-brain tissue using a hybrid watershed/surface deformation procedure, automated Talairach transformation, and segmentation of the subcortical white matter and deep grey matter volumetric structures, intensity correction and delineation of grey/ white/cerebrospinal fluid boundaries. Following completion of cortical models a number of deformable procedures were applied including surface inflation, registration to a spherical atlas (Dale et al. 1999) and parcellation of the cerebral cortex into neuroanatomical units based on gyral and sulcal structure (Desikan et al. 2006). The segmentation and parcellation processes use probabilistic information estimated from the manually labelled training set to assign a neuroanatomical label automatically to each voxel.

\section{Regions of interest}

Total brain volume (TBV) and cortical GM volumes were considered as global measures of cerebral health while HC and DMN were considered as specific ROIs known to be implicated in the AD pathological process (Simic et al. 2014; Grothe and Teipel 2016; Chang et al. 2015; Koch et al. 1991). The volumes of structures of the left and right DMN were computed by summing the volumes of their subcomponents (see (Buckner et al. 2008; Laird et al. 2009; Andrews-Hanna et al. 2010; Thomas Yeo et al. 2011) for a rationale of this selection) including the Medial OrbitoFrontal (MOF), Entorhinal (ERC), Para-Hippocampal (P-HC), Fusiform (FUS), Posterior Cingulate (PCC), Isthmus Cingulate (ICC), PreCuneus (PCU) cortices and the Inferior Parietal Lobule (IPL) (Fig. 1).

\section{ANU Alzheimer's Disease Risk Index (ANU-ADRI)}

The ANU-ADRI (http://anuadri.anu.edu.au) is a questionnaire-based instrument which has been developed to estimate future risk of developing AD. To ensure its robustness it has been constructed based on risk estimates from published meta-analyses of established risk factors which can be assessed by self-report. The methodology used in its development is discussed and published elsewhere (Anstey et al. 2013). Briefly, it assesses up to 15 domains (age, sex, education, BMI, depression, diabetes, cholesterol, smoking, traumatic brain injury, physical activity, cognitive activity, social engagement, alcohol intake, dietary fish intake, pesticide exposure) and produces corresponding risk sub-scores by allocating points (weighted relative to each risk factor's effect size) for varying levels of each domain reported by individual users. An overall composite score is computed by summing all available sub-scores. The ANU-ADRI has been validated against three large international studies and was found to be reliable in estimating one's risk of developing $\mathrm{AD}$ and to be robust when not all risk measures are available for its computation (Anstey et al. 2014). In the present dataset, 11 available risk measures were considered for analysis including age, sex, education, depression, diabetes, smoking, traumatic brain injury, physical activity, cognitive activity, social engagement, alcohol intake. Note that while a BMI measure was also available it was not included as current evidence from meta-analysis indicates a risk for this factor is only present in mid-life.

\section{Diagnosis and cognitive change}

Participants' cognitive status was assessed at the first wave of assessment and re-assessed approximately every 4 years over a follow-up of 12 years (waves 2-4). At waves 1 to 3 diagnoses were assessed in a two-step process. Participants were first assessed on a number of cognitive measures including the MMSE, immediate and delayed recall, symboldigit substitution test, simple and complex reaction time, and Purdue pegboard) as part of the main survey. If they performed below specific thresholds [any one of the following (1) a MMSE score $\leq 25$; (2) a score below the 5th percentile on immediate or delayed recall, or (3) a score below the 5th percentile on one of the following tests: symbol-digit substitution test or Purdue Pegboard with both hands or reaction time] they were administered a full neuropsychological
Fig. 1 Sub-regions of the Default Mode Network (DMN)

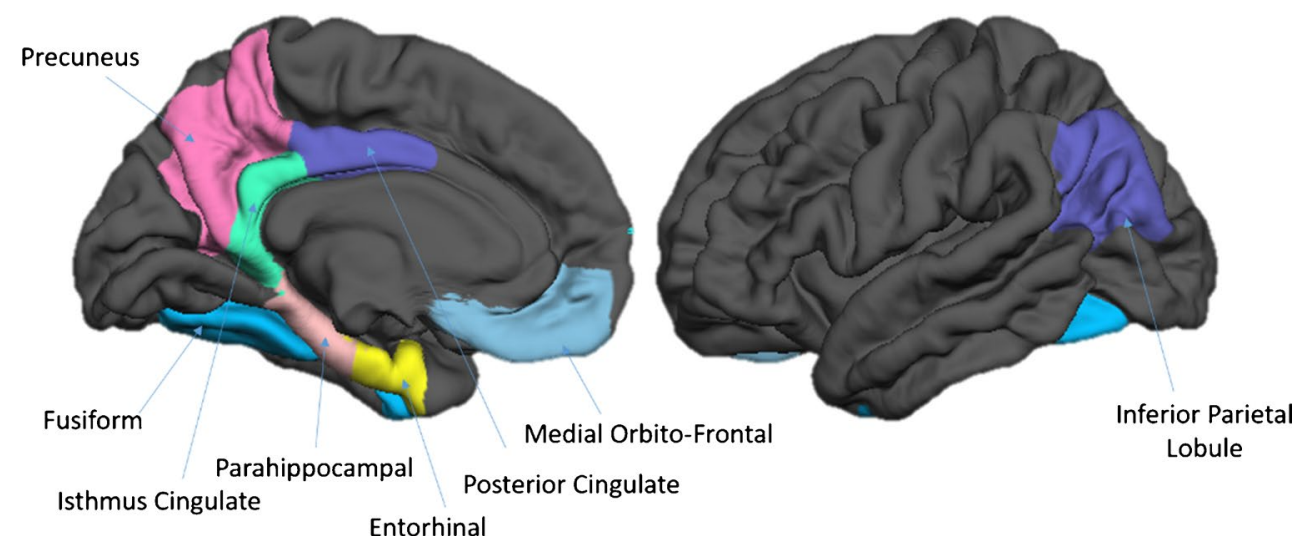

Entorhinal 
assessment on which a consensus diagnosis was established based on published criteria for MCI (Petersen/Winblad) and dementia (DSM-IV) (Petersen et al. 1999; Winblad et al. 2004). At the fourth wave, because of the higher prevalence of MCI and dementia in this age group, all participants were administered a neuropsychological assessment and given a diagnosis if they met clinical criteria. As an additional measure of cognitive decline a change score was computed between the latest MMSE score and the baseline score to reflect change in general cognitive functioning over the follow-up period.

\section{Statistical analysis}

Statistical analyses were computed in the R statistical package (version 3.1). Descriptive analyses were conducted using Chi-square tests for categorical data and t-tests to compare groups on continuous variables. Cross-sectional associations between ANU-ADRI risk scores and TBV, cortical GM, HC and DMN structures volume at first assessment were investigated with linear regression analyses controlling for sex, age, intra-cranial volume (ICV) and APO*E4 genotype. Followup analyses were conducted in each hemisphere to identify topographical differences. Variables which did not follow a normal distribution were transformed with an appropriate function (log). Multivariate outliers were excluded if their Mahalanobis distance was greater than $13.82(p<0.001)$, leading to a maximum of 4 participants being excluded for any single ROI. Cox proportional hazard ratios were computed to test the associations between ANU-ADRI score, DMN volume and risk of cognitive impairment (MCI/ dementia) or change in MMSE score over the follow-up ( $\mathrm{R}$ package "survival") with time to diagnosis as time metric. Alpha was set at 0.05 with Bonferroni correction.

\section{Results}

The participants' demographic measures are presented in Table 1. Male were not substantially different from female participants on most characteristics except form men being more likely to be hypertensive and for women having a slightly lower BMI and fewer years of education, as well as engaging somewhat less in physical and cognitive activity. The total ANU-ADRI scores ranged from -18 to +10 with a mean of -8.20 (SD 5.67) indicating that on average participants were exposed to more protection than risk given their profile. Left GM (\%) and HC (\%) volumes were larger than right volumes. The volume of DMN structures was larger in the right than left hemisphere $(\sim 1 \%)$ and was composed of MOF $(\sim 11.2 \%)$, ERC $(4.4 \%)$, P-HC ( 4.8\%), FUS $(\sim 20.9 \%)$, PCC $(\sim 6.7 \%)$, ICC $(\sim 5.5 \%)$, IPL $(\sim 27.3 \%)$, and PCU $(19.2 \%)$ with each contributing a similar proportion of variance to the overall volume in the DMN. Histogram, scatter, and boxplots showing the distribution of left and right cortical gray matter, hippocampal and DMN structure volumes and their association with the total ANU-ADRI score are presented in Fig. 2.

\section{Associations between ANU-ADRI scores and DMN volume}

Analyses investigating the relationship between ANU-ADRI scores and ROIs while controlling for age, sex, ICV and APO*E4 genotype revealed significant negative association with left and right cortical gray matter, and left and right DMN (Table 2). This indicated that for every one point increase in the ANU-ADRI there was an approximately $0.25 \%$ and $0.32 \%$ decrease in cortical and DMN structure volume respectively, which translates to an 7-9\% difference in volume in these regions between those with the lowest and highest risk scores. No significant associations were detected with TBV, left or right hippocampus.

Follow-up analyses testing regional differences within the left and right DMNs are presented in Table 3. Significant associations were detected between ANU-ADRI scores and the P-HC, FUS, ICC, and IPL in the two hemispheres and with the left MOF. Trends were also detected for right MOF and PCC.

\section{ANU-ADRI score, DMN, and risk of cognitive impairment}

Although the ANU-ADRI has been validated in three large international datasets and its association with increased risk of MCI has been demonstrated within the larger PATH cohort (Andrews et al. 2017), it was deemed important to confirm its predictive value for $\mathrm{MCI} /$ dementia within the sample studied here. Of the participants included at baseline and without any cognitive impairment 3 participants developed dementia ( 2 of those were previously diagnosed with MCI), 51 developed MCI (including the two who were subsequently diagnosed with dementia), and 409 remained cognitively healthy over the 12-year follow-up. Cox proportional hazard ratio analyses revealed that the ANU-ADRI score was significantly associated with conversion to $\mathrm{MCI} / \mathrm{demen}$ tia (HR 1.08, 95\% CI 1.03-1.13, $p=0.002)$ after controlling for age, sex and APO*E4 genotype. Of the five DMN ROIs (volumes transformed into $\mathrm{ml}$ to facilitate interpretation) which were significantly predicted by the ANU-ADRI score, the left MOF was the only region found to be associated with an increased risk of developing MCI/dementia (HR $0.61,95 \%$ CI $0.04-0.92, p=0.018$ ). This indicates that for every additional $1 \mathrm{ml}$ in MOF volume at baseline the risk of cognitive impairment over the follow-up decreased by $64 \%$. Finally, a formal mediation analysis (Baron and Kenny 
Table 1 Participants' demographic characteristics

\begin{tabular}{|c|c|c|c|c|}
\hline Measures & Whole sample $(n=461)$ & Males $(n=238)$ & Females $(n=223)$ & T/chi-sq test ( $\mathrm{P}$ value) \\
\hline Age, years (SD) & $63.05(1.44)$ & $63.10(1.42)$ & $63.00(1.47)$ & $0.78(0.437)$ \\
\hline Education, years (SD) & $13.99(2.62)$ & $14.39(2.57)$ & $13.56(2.60)$ & $3.44(0.001)$ \\
\hline MMSE, score (SD) & $29.34(0.90)$ & $29.29(0.92)$ & $29.41(0.87)$ & $-1.46(0.144)$ \\
\hline BMI, kg/m2 (SD) & $26.53(4.31)$ & $26.58(3.47)$ & $26.47(5.07)$ & $0.27(0.791)$ \\
\hline Hypertension, n (\%) & $285(61.82 \%)$ & $160(67.23 \%)$ & $125(56.05 \%)$ & $5.63(0.018)$ \\
\hline $\mathrm{APO} * \mathrm{E} 4, \mathrm{n}(\%)$ & $123(26.68 \%)$ & $65(27.31 \%)$ & $58(26.01 \%)$ & $0.04(0.833)$ \\
\hline Alcohol, score (SD) & $-2.48(1.14)$ & $-2.55(1.08)$ & $-2.41(1.20)$ & $-1.30(0.194)$ \\
\hline Education, score (SD) & $0.61(1.30)$ & $0.50(1.16)$ & $0.73(1.44)$ & $-1.82(0.069)$ \\
\hline Cognitive activity, score (SD) & $-4.92(2.86)$ & $-5.26(2.62)$ & $-4.56(3.05)$ & $-2.65(0.008)$ \\
\hline Depression, score (SD) & $0.05(0.32)$ & $0.03(0.26)$ & $0.07(0.37)$ & $-1.27(0.205)$ \\
\hline Diabetes, score (SD) & $0.23(0.81)$ & $0.26(0.85)$ & $0.20(0.75)$ & $0.84(0.401)$ \\
\hline Education, score (SD) & $0.61(1.30)$ & $0.50(1.16)$ & $0.73(1.44)$ & $-1.82(0.069)$ \\
\hline Physical activity, score (SD) & $-1.06(1.17)$ & $-1.22(1.19)$ & $-0.89(1.13)$ & $-3.05(0.002)$ \\
\hline Smoking, score (SD) & $0.64(1.06)$ & $0.70(1.01)$ & $0.57(1.10)$ & $1.29(0.197)$ \\
\hline Social engagement, score (SD) & $0.72(1.51)$ & $0.59(1.37)$ & $0.85(1.64)$ & $-1.86(0.063)$ \\
\hline TBI, score (SD) & $0.17(0.82)$ & $0.27(1.00)$ & $0.07(0.53)$ & $2.66(0.008)$ \\
\hline ANU-ADRI, score (SD) & $-8.20(5.67)$ & $-8.69(5.65)$ & $-7.68(5.66)$ & $-1.93(0.054)$ \\
\hline $\mathrm{TBV}, \mathrm{mm}^{3}(\mathrm{SD})$ & $1150449.66(126363.06)$ & $1222160.89(115338.48)$ & $1073914.81(86528.32)$ & $15.67(0.000)$ \\
\hline Left GM, mm³ (SD) & 209832.88 (24694.96) & 221804.07 (24817.49) & $197056.46(16985.34)$ & $12.56(0.000)$ \\
\hline Right GM, mm³ (SD) & 210880.75 (23986.38) & $222450.90(23761.95)$ & 198532.35 (17108.24) & $12.46(0.000)$ \\
\hline Left DMN, mm³ (SD) & $35339.57(4140.20)$ & 37413.33 (3993.63) & $33126.31(2993.35)$ & $13.09(0.000)$ \\
\hline Right DMN, mm³ (SD) & $36531.44(4360.38)$ & $38696.23(4186.08)$ & $34221.04(3209.80)$ & $12.93(0.000)$ \\
\hline Left MOF, $\mathrm{mm}^{3}(\mathrm{SD})$ & $5057.98(772.34)$ & $5382.60(788.12)$ & $4711.53(582.54)$ & $10.44(0.000)$ \\
\hline Right MOF, $\mathrm{mm}^{3}$ (SD) & $4910.17(654.92)$ & $5176.12(651.75)$ & $4626.32(527.74)$ & $9.98(0.000)$ \\
\hline Left ERC, $\mathrm{mm}^{3}(\mathrm{SD})$ & $2044.63(372.38)$ & $2176.18(371.29)$ & $1904.23(319.05)$ & $8.45(0.000)$ \\
\hline Right ERC, $\mathrm{mm}^{3}$ (SD) & $1833.20(372.47)$ & $1942.74(387.51)$ & $1716.29(317.12)$ & $6.88(0.000)$ \\
\hline Left P-HC, mm $^{3}$ (SD) & $2195.33(363.03)$ & $2282.86(412.63)$ & $2101.91(272.65)$ & $5.59(0.000)$ \\
\hline Right P-HC, mm ${ }^{3}$ (SD) & $2089.20(384.64)$ & $2153.98(397.00)$ & $2020.06(359.18)$ & $3.80(0.000)$ \\
\hline Left FUS, $\mathrm{mm}^{3}$ (SD) & $9478.72(1384.42)$ & $9959.11(1369.72)$ & 8966.01 (1207.05) & $8.27(0.000)$ \\
\hline Right FUS, mm³ (SD) & 9107.03 (1393.85) & 9681.33 (1374.17) & $8494.11(1130.83)$ & $10.15(0.000)$ \\
\hline Left PCC, $\mathrm{mm}^{3}$ (SD) & $2952.70(505.92)$ & $3148.05(510.80)$ & 2744.21 (408.93) & $9.40(0.000)$ \\
\hline Right PCC, $\mathrm{mm}^{3}$ (SD) & 3027.73 (515.74) & $3196.79(515.06)$ & $2847.29(452.33)$ & $7.75(0.000)$ \\
\hline Left ICC, $\mathrm{mm}^{3}$ (SD) & $2512.00(453.11)$ & $2681.97(480.33)$ & $2330.61(339.25)$ & $9.12(0.000)$ \\
\hline Right ICC, $\mathrm{mm}^{3}$ (SD) & 2340.64 (411.27) & $2435.37(427.60)$ & $2239.52(367.95)$ & $5.28(0.000)$ \\
\hline Left IPL, $\mathrm{mm}^{3}(\mathrm{SD})$ & 11098.21 (1741.79) & $11782.56(1777.59)$ & $10367.82(1369.32)$ & $9.61(0.000)$ \\
\hline Right IPL, mm³ (SD) & $13223.48(2057.40)$ & $14109.90(2059.66)$ & $12277.43(1581.33)$ & $10.75(0.000)$ \\
\hline
\end{tabular}

Scoring of the ANU-ADRI sub-components (i.e. alcohol, cognitive activity, depression, diabetes, education, physical activity, smoking, social engagement, traumatic brain injury) is presented in detail in (Anstey 2013)

$T B I$ traumatic brain injury, $T B V$ total brain volume, $G M$ gray matter, $W M$ white matter, $D M N$ default mode network, $M O F$ medial orbito-frontal, $E R C$ entorhinal, $P$-HC para-hippocampal, $F U S$ fusiform, $P C C$ posterior cingulate, $I C C$ isthmus cingulate, $P C U$ precuneus cortices, $I P L$ inferior parietal lobule

Significance: $p<0.05$

1986) was conducted to determine whether the risk of developing MCI/dementia associated with a higher ANU-ADRI score was mediated by left MOF volume. It revealed a partial mediation as the HR and significance associated with the ANU-ADRI score (HR 1.07, 95\%CI 1.02-1.13, $p=0.005$ ) were significantly reduced (Chi square $=7.27, p=0.03$ ) when MOF volume was controlled for in the analysis. None of the total left or right DMN and left or right GM volumes significantly predicted $\mathrm{MCI} /$ dementia (results not shown) and therefore mediation analyses for these structures could not be conducted.

The associations between ANU-ADRI score and baseline MMSE score and change in MMSE score over the follow-up were also tested and revealed significant effects 
Fig. 2 Distribution of left and right volume of structures in the Default Mode Network (left), and scatter plot of the association between ANU-ADRI total scores and volumes in the DMN (right)
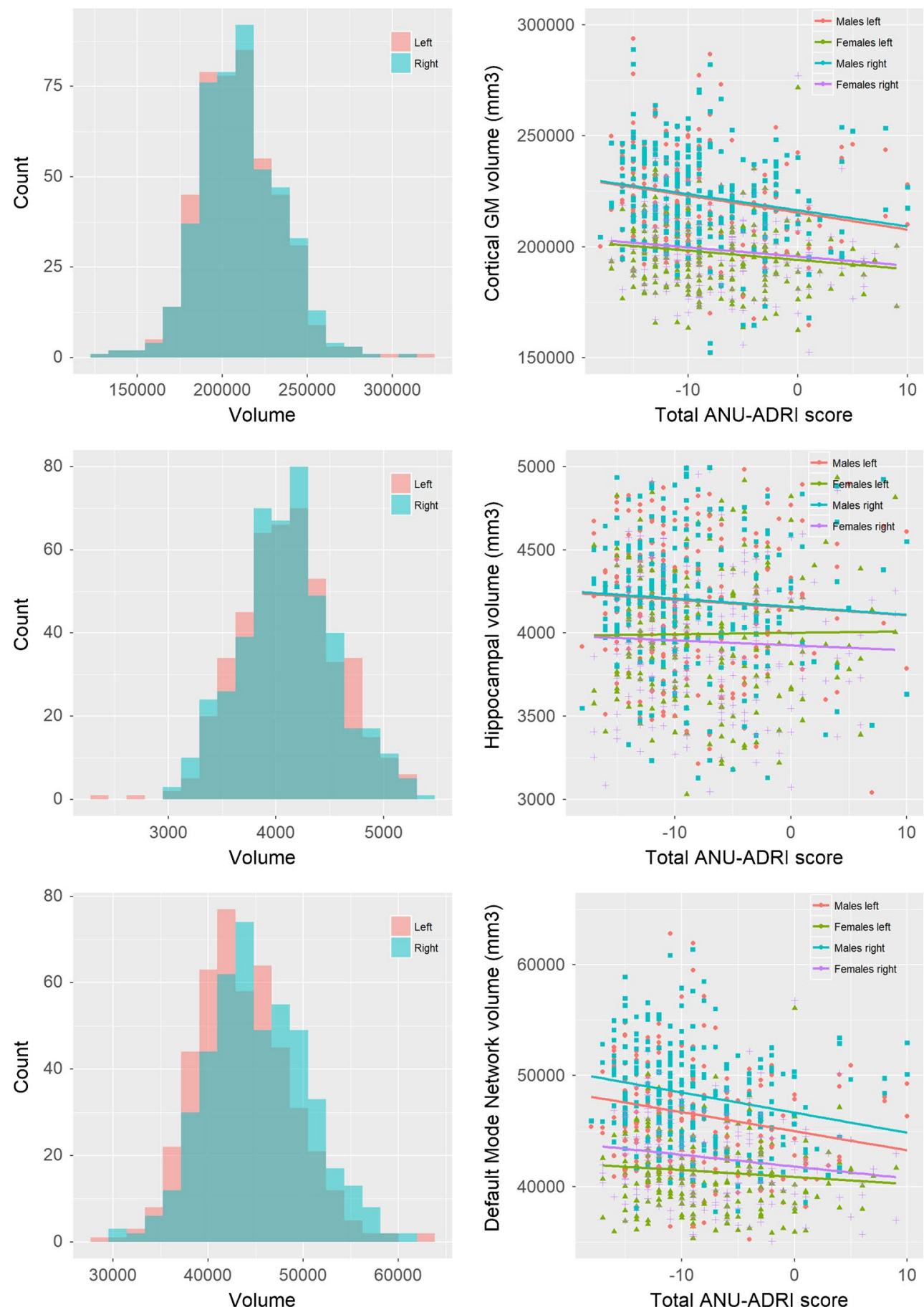

(Baseline MMSE estimate: $-0.038, p=0.007$; MMSE change estimate: $-0.025, p=0.012$ ) indicating that those in the lower ANU-ADRI quartile (less risk) would be predicted to have a 0.53 point higher MMSE score at baseline and a 0.35 point lower decrease in MMSE score over the follow-up than those in the upper quartile (given 14 points difference between first and last ANU-ADRI quartiles).

\section{Sensitivity analyses}

All analyses were controlled for APO*E4 genotype to ensure the effects demonstrated occur above and beyond the risk imparted by the main AD genetic risk factor. However, to determine whether significant shared variance explained part of the relationship between ANU-ADRI score, volumes of 
Table 2 Associations between ANU-ADRI scores and ROIs volumes assessed by multiple linear regression analyses and controlling for age, sex, ICV and APO*E4 genotype. Significance levels are reported after Bonferoni corrections

\begin{tabular}{llllllll}
\hline & TBV & Left GM & Right GM & Left HC & Right HC & Left DMN & Right DMN \\
\hline ANU-ADRI & 246.29 & $-542.80^{* * *}$ & $-557.86^{* * *}$ & -0.92 & -4.46 & $-131.41^{* * *}$ & $-162.19^{* * *}$ \\
& $p=0.525$ & $p<0.001$ & $p<0.001$ & $p=0.782$ & $p=0.170$ & $p<0.001$ & $p<0.001$ \\
Sex & $-23,809.82^{* * *}$ & $-10,914.52^{* * *}$ & $-10,423.89^{* * *}$ & -70.98 & $-116.21^{* *}$ & $-2,184.91^{* * *}$ & $-2,446.86^{* * *}$ \\
& $p<0.001$ & $p<0.001$ & $p<0.001$ & $p=0.111$ & $p=0.008$ & $p<0.001$ & $p<0.001$ \\
Age & $-4,589.17^{* *}$ & $-1,545.40^{* *}$ & $-1,910.45^{* * *}$ & $-37.50^{* *}$ & $-32.83^{*}$ & $-306.71^{* *}$ & $-396.22^{* *}$ \\
& $p=0.003$ & $p=0.005$ & $p<0.001$ & $p=0.005$ & $p=0.011$ & $p=0.009$ & $p=0.002$ \\
ICV & $0.62^{* * *}$ & $0.06^{* * *}$ & $0.06^{* * *}$ & $0.01^{* * *}$ & $0.01^{* * *}$ & $0.01^{* * *}$ & $0.01^{* * *}$ \\
& $p<0.001$ & $p<0.001$ & $p<0.001$ & $p<0.001$ & $p<0.001$ & $p<0.001$ & $p<0.001$ \\
APO*E4 & $3,333.17$ & 522.79 & $1,412.52$ & 19.20 & 14.57 & 177.26 & 397.37 \\
& $p=0.499$ & $p=0.770$ & $p=0.424$ & $p=0.651$ & $p=0.725$ & $p=0.638$ & $p=0.316$ \\
Constant & $486,015.10^{* * *}$ & $209,495.20^{* * *}$ & $233,341.60^{* * *}$ & $5,201.03^{* * *}$ & $4,792.94^{* * *}$ & $41,687.87^{* * *}$ & $47,935.97^{* * *}$ \\
& $p<0.001$ & $p<0.001$ & $p<0.001$ & $p<0.001$ & $p<0.001$ & $p<0.001$ & $p<0.001$ \\
Adjusted R & 0.856 & 0.482 & 0.484 & 0.173 & 0.228 & 0.489 & 0.498 \\
Residual Std. error & $46,624.82$ & $16,871.34$ & $16,698.09$ & 401.010 & 391.94 & $3,558.79$ & $3,752.08$ \\
F statistic & $544.57^{* * *}$ & $86.03^{* * *}$ & $86.67^{* * *}$ & $20.13^{* * *}$ & $28.16^{* * *}$ & $88.78^{* * *}$ & $92.02^{* * *}$ \\
& $(\mathrm{df}=5 ; 454)$ & $(\mathrm{df}=5 ; 452)$ & $(\mathrm{df}=5 ; 452)$ & $(\mathrm{df}=5 ; 452)$ & $(\mathrm{df}=5 ; 454)$ & $(\mathrm{df}=5 ; 454)$ & $(\mathrm{df}=5 ; 454)$ \\
\hline
\end{tabular}

Significance: $* p<0.05, * * p<0.01, * * * p<0.001$

DMN structures, and cognitive functioning, all analyses were repeated without controlling for APO*E4 genotype. Results (not shown) remained almost identical suggesting that the risk indexed by the ANU-ADRI explains variance in outcome measures unrelated to APO*E4.

\section{Discussion}

This study's main findings are that greater exposure to risk factors associated with the development of $\mathrm{AD}$, as assessed with the ANU-ADRI, in community living participants in their early 60s is associated with lower brain volumes (cortical GM and DMN), a greater risk of experiencing decline in general cognitive functioning later in life, and a greater risk of developing MCI.

Higher scores on the ANU-ADRI were associated with lower volumes in the left and right cortical GM and DMN. This effect was very substantial with every additional point being associated with a $0.32 \%$ lower volume of DMN structures which equates to a $9 \%$ difference in volume across the range of scores observed in this sample. In addition, risk scores were differentially associated with sub-regions of the DMN with effects being greatest for the left MOF $(0.3 \% /$ pt), left and right P-HC (0.29/0.31\%/pt), left and right FUS $(0.46 / 0.47 \% / \mathrm{pt})$, left and right ICC $(0.29 / 0.33 \% / \mathrm{pt})$, and left and right IPL $(0.27 / 0.39 \% / p t)$. It is also noteworthy that all DMN sub-regions were negatively associated with the risk score whether significantly so or not.
Importantly, ANU-ADRI risk scores also predicted baseline general cognitive functioning, with each additional risk point being associated with a $0.14 \%$ lower MMSE score, as well as change in function $(-0.13 \% / \mathrm{pt}$ for each additional year of follow-up). This is significant as for a person with a risk score of 5 (compared to a score of 0 with no risk and no protection) it would correspond to a $6.5 \%$ lower MMSE over a 10 year period. Consistent with these findings results also showed that higher risk scores were associated with an $8 \%$ increased risk of developing $\mathrm{MCI} / \mathrm{dementia}$ over the 12-year follow-up.

To test the hypothesis that DMN structures mediate the relationship between risk scores and cognitive impairment further analyses investigating the association between DMN sub-regions shown to be significantly predicted by ANUADRI scores and risk of developing $\mathrm{MCI} /$ dementia were conducted. They revealed that for every $1 \%$ larger MOF volume there was a $3.2 \%$ decreased risk of developing MCI/ dementia over the follow-up. Importantly, the subsequent mediation analysis showed that the effect linking ANUADRI risk score level and risk of developing $\mathrm{MCI} /$ dementia was partially mediated by the left MOF (but not not by total $\mathrm{GM}$ ) and thus supports a theoretical link between risk exposure, brain structure in the DMN, and cognitive impairment.

Importantly, all analyses were controlled for $\mathrm{APO} * \mathrm{E} 4$ genotype which could potentially explain both the difference in volume and the difference in cognition observed. Moreover, additional sensitivity analyses which did not control for APO*E4 genotype indicated that the present effect were neither partially masked nor explained by this AD genetic risk 


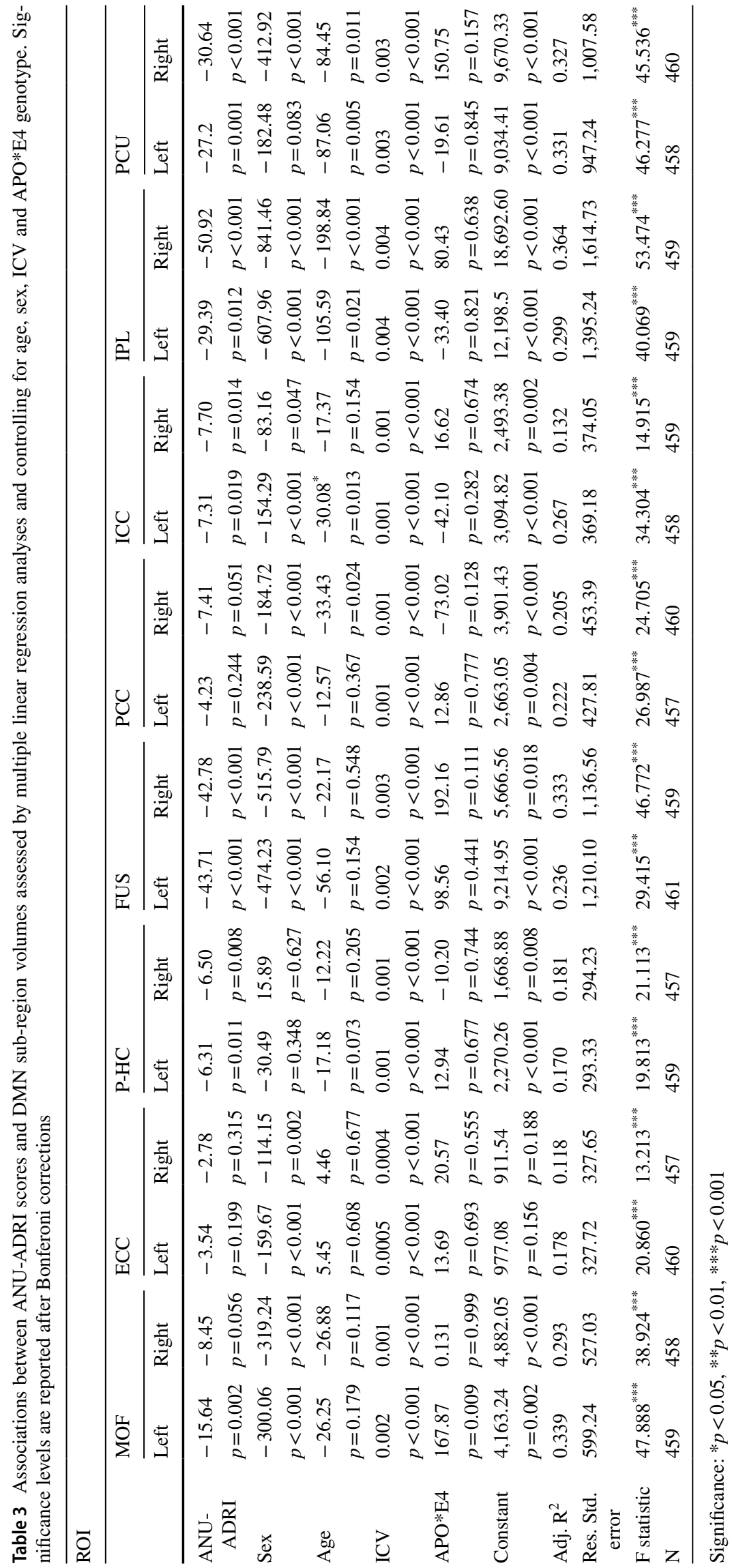


factor. In fact, somewhat surprisingly, APO*E4 status did not explain any of the outcome variable except for the left MOF where being a carrier was associated contrary to what might have been expected, with a 3.3\% increased volume. This is consistent with our previous research showing that the ANU-ADRI is a stronger predictors of cognitive decline than a composite genetic index including APO*E4 and other $\mathrm{AD}$ risk genes (Andrews et al. 2017).

A question arising from these findings is whether the variability in DMN volume related to risk estimated with the ANU-ADRI is specific to AD pathology or more generally related to the vulnerability of the DMN to a range of assaults due to its high and sustained metabolism, even at rest, relative to other brain networks. On one hand it is clear that a number of factors contributing to the ANU-ADRI score are also associated with increased amyloid plaque formation which would suggest that risk estimates are relatively specific to AD pathology (Luciano et al. 2015; He et al. 2017; Johnson et al. 2010). On the other hand, these same factors are associated with broad pathological mechanisms including systemic inflammation, oxidative stress, atherosclerosis, and impaired cardio-metabolic function which may have a less specific impact on the DMN via small vessel disease, chronic hypo-perfusion, micro-infarcts, and shrinking neuronal processes. However, given that up to $80 \%$ of $\mathrm{AD}$ cases have comorbid vascular disease (Attems and Jellinger 2014) this distinction may be moot. Nevertheless, it would be interesting for future studies to investigate whether ANU-ADRI estimates are specifically related to incident amyloid plaque formation.

Overall, these findings are important because they show that exposure to modifiable risk factors, when assessed with a valid instrument, is significantly associated with lower volumes in a brain network known to be particularly strongly affected in AD thus confirming previously demonstrated associations between risk factor exposure and risk of cognitive impairment. As such this study adds to the evidence base necessary to recommend to individuals living in the community that they decrease their risk exposure. It also indicates that behavior optimization and targeted preventative interventions should occur at mid-life or preferably before as the pathological processes mediating the effect of modifiable risk factors are likely to develop progressively over decades. These findings are also encouraging because they suggest that identifying individuals at risk on a large scale in a population is feasible since the ANU-ADRI can be administered online and has already been implemented in a free web-portal accessible by all (http://anuadri.anu.edu.au). Thus, those involved in recruiting participants for behavioral or pharmacological studies may want to consider the ANUADRI as an effective and inexpensive screening method.

This research has many strengths including a large sample size for a neuroimaging study, both brain and cognitive outcomes, the use of a validated measure of risk exposure and clear theoretically based hypotheses. However, it also has a number of limitations. Relationships between ANUADRI risk scores and brain were estimated with crosssectional analyses and therefore do not permit causal interpretations. The ANU-ADRI scores were computed based on a sub-set of risk factors and while our previous validation studies have shown that the measure was robust to inclusion of a variable number of risk factors, availability of all included risk factors may have provided more accurate estimates and more powerful predictions. Finally, this investigation focused on a narrow age range which has the benefit of reducing cohort effects but may produce findings not be completely applicable to other age groups.

In summary, the evidence on modifiable risk factors is strong, screening for individuals at risk is achievable in general, but is made much more accessible through instruments such as the ANU-ADRI. This and other confirmatory studies provide confidence on the relevance, validity, and usefulness of this tools. A stronger focus needs to be put on behavior change to achieve risk reduction in the population.

Acknowledgements The authors are grateful to Anthony Jorm, Helen Christensen, Peter Butterworth, Andrew McKinnon, and the PATH project interviewers. This research was partly undertaken on the National Computational Infrastructure (NCI) facility in Canberra, Australia, which is supported by the Australian Commonwealth Government.

Author contributions Assoc. Prof. Cherbuin contributed to the design of the study, conducted all statistical analyses, and managed all aspects of manuscript preparation and submission. Dr. Shaw provided theoretical expertise, conducted part of the neuroimaging analyses, and contributed to writing and editing the manuscript. Dr. Walsh provided theoretical expertise, contributed to data preparation and statistical analyses, and contributed to writing and editing the manuscript. Prof. Sachdev provided methodological input and theoretical expertise, and contributed to writing and editing of the manuscript. Prof. Anstey contributed to the design of the study, provided methodological input and theoretical expertise, and contributed to writing and editing of the manuscript.

Funding The study was supported by NHMRC Grant 973302,179805 , 157125, 1063907. Nicolas Cherbuin is funded by ARC Future Fellowship no. 120100227 and Kaarin Anstey by NHMRC Fellowship 1002560.

\section{Compliance with ethical standards}

Disclosures Assoc. Prof. Cherbuin reports no disclosures. Dr Shaw reports no disclosures. Dr Walsh reports no disclosures. Prof. Sachdev reports no disclosures. Prof. Anstey reports no disclosures.

Conflict of interest All authors declare that they have no conflict of interest.

Ethical approval All procedures performed in studies involving human participants were in accordance with the ethical standards of (and 
approved by) the Australian National University Ethics committee and with the 1964 Helsinki declaration and its later amendments or comparable ethical standards.

Informed consent Informed consent was obtained from all individual participants included in the study.

Open Access This article is distributed under the terms of the Creative Commons Attribution 4.0 International License (http://creativecommons.org/licenses/by/4.0/), which permits unrestricted use, distribution, and reproduction in any medium, provided you give appropriate credit to the original author(s) and the source, provide a link to the Creative Commons license, and indicate if changes were made.

\section{References}

Andrews, S. J., Eramudugolla, R., Velez, J., Cherbuin, N., Easteal, S., \& Anstey, K. J. (2017). Validating the role of the Australian National University Alzheimer's disease risk index (ANU-ADRI) and a genetic risk score in progression to cognitive impairment in a population-based cohort of older adults followed for 12 years. Alzheimer's Research \& Therapy, 9(1), 16.

Andrews-Hanna, J. R., Reidler, J. S., Sepulcre, J., Poulin, R., \& Buckner, R. L. (2010). Functional-anatomic fractionation of the brain's default network. Neuron, 65, 550-562.

Anstey, K. J., Cherbuin, N., \& Herath, P. M. (2013). Development of a new method for assessing global risk of Alzheimer's disease for use in population health approaches to prevention. Prevention Science, 14, 411-421.

Anstey, K. J., Cherbuin, N., Herath, P. M., et al. (2014). A self-report risk index to predict occurrence of dementia in three independent cohorts of older adults: the ANU-ADRI. PloS One, 9, e86141.

Anstey, K. J., Christensen, H., Butterworth, P., et al. (2012). Cohort profile: the PATH through life project. International Journal of Epidemiology, 41, 951-960.

Attems, J., \& Jellinger, K. A. (2014). The overlap between vascular disease and Alzheimer's disease-lessons from pathology. BMC Medicine, 12, 206.

Baron, R. M., \& Kenny, D. A. (1986). The moderator-mediator variable distinction in social psychological research: conceptual, strategic, and statistical considerations. Journal of Personality and Social Psychology, 51, 1173-1182.

Buckner, R. L., Andrews-Hanna, J. R., \& Schacter, D. L. (2008). The brain's default network: anatomy, function, and relevance to disease. Annals of the New York Academy of Sciences, 1124, 1-38.

Chang, Y. T., Huang, C. W., Chang, Y. H., et al. (2015). Amyloid burden in the hippocampus and default mode network: relationships with gray matter volume and cognitive performance in mild stage Alzheimer disease. Medicine, 94, e763.

Dale, A. M., Fischl, B., \& Sereno, M. I. (1999). Cortical surface-based analysis. I. Segmentation and surface reconstruction. NeuroImage, 9, 179-194.

Desikan, R. S., Segonne, F., Fischl, B., et al. (2006). An automated labeling system for subdividing the human cerebral cortex on MRI scans into gyral based regions of interest. NeuroImage, 31, 968-980.

Fischl, B. (2012). FreeSurfer. Neuroimage, 62, 774-781.

Fjell, A. M., McEvoy, L., Holland, D., Dale, A. M., \& Walhovd, K. B. (2014). What is normal in normal aging? Effects of aging, amyloid and Alzheimer's disease on the cerebral cortex and the hippocampus. Progress in Neurobiology, 117, 20-40.
Goldberg, D., Bridges, K., Duncan-Jones, P., \& Grayson, D. (1988). Detecting anxiety and depression in general medical settings. BMJ, 297, 897-899.

Grothe, M. J., \& Teipel, S. J. (2016). Spatial patterns of atrophy, hypometabolism, and amyloid deposition in Alzheimer's disease correspond to dissociable functional brain networks. Human Brain Mapping, 37, 35-53.

He, X-f, Liu, D-x, Zhang, Q., et al. (2017). Voluntary exercise promotes glymphatic clearance of amyloid beta and reduces the activation of astrocytes and microglia in aged mice. Frontiers in Molecular Neuroscience, 10, 144.

Johnson, V. E., Stewart, W., \& Smith, D. H. (2010). Traumatic brain injury and amyloid-beta pathology: a link to Alzheimer's disease? Nature Reviews Neuroscience, 11, 361-370.

Koch, K., Myers, N. E., Gottler, J., et al. (2015). Disrupted intrinsic networks link amyloid-beta pathology and impaired cognition in prodromal Alzheimer's disease. Cerebral Cortex (New York, NY: 1991), 25, 4678-4688.

Laird, A. R., Eickhoff, S. B., Li, K., Robin, D. A., Glahn, D. C., \& Fox, P. T. (2009). Investigating the functional heterogeneity of the default mode network using coordinate-based meta-analytic modeling. The Journal of Neuroscience : the Official Journal of the Society for Neuroscience, 29, 14496-14505.

Luciano, R., Barraco, G. M., Muraca, M., et al. (2015). Biomarkers of Alzheimer disease, insulin resistance, and obesity in childhood. Pediatrics, 135, 1074-1081.

Mormino, E. C., Smiljic, A., Hayenga, A. O., et al. Relationships between beta-amyloid and functional connectivity in different components of the default mode network in aging. Cerebral Cortex (New York, NY: 1991) 2011;21:pp. 2399-2407.

Petersen, R. C., Smith, G. E., Waring, S. C., Ivnik, R. J., Tangalos, E. G., \& Kokmen, E. (1999). Mild cognitive impairment: clinical characterization and outcome. Archives of Neurology, 56, 303-308.

Petrella, J. R., Sheldon, F. C., Prince, S. E., Calhoun, V. D., \& Doraiswamy, P. M. (2011). Default mode network connectivity in stable vs progressive mild cognitive impairment. Neurology, 76, 511-517.

Sheline, Y. I., Raichle, M. E., Snyder, A. Z., et al. (2010). Amyloid plaques disrupt resting state default mode network connectivity in cognitively normal elderly. Biological Psychiatry, 67, 584-587.

Simic, G., Babic, M., Borovecki, F., \& Hof, P. R. (2014). Early failure of the default mode network and the pathogenesis of Alzheimer's disease. CNS Neuroscience \& Therapeutics, 20, 692-698.

Sled, J. G., Zijdenbos, A. P., \& Evans, A. C. (1998). A nonparametric method for automatic correction of intensity nonuniformity in MRI data. IEEE Transactions on Medical Imaging, 17, 87-97.

Thomas Yeo, B. T., Krienen, F. M., Sepulcre, J., et al. (2011). The organization of the human cerebral cortex estimated by intrinsic functional connectivity. Journal of Neurophysiology, 106, $1125-1165$.

Tondelli, M., Wilcock, G. K., Nichelli, P., De Jager, C. A., Jenkinson, M., \& Zamboni, G. (2012). Structural MRI changes detectable up to ten years before clinical Alzheimer's disease. Neurobiology of Aging, 33, 825.e825-836.

Winblad, B., Palmer, K., Kivipelto, M., et al. (2004). Mild cognitive impairment-beyond controversies, towards a consensus: report of the International Working Group on Mild Cognitive Impairment. Journal of Internal Medicine, 256, 240-246.

$\mathrm{Xu}$, W., Tan, L., Wang, H.-F., et al. (2015). Meta-analysis of modifiable risk factors for Alzheimer's disease. Journal of Neurology, Neurosurgery \& Psychiatry, 86, 1284-1285. 